\title{
Descripción anatómica de la irrigación del colgajo sural a pedículo distal en piezas cadavéricas*
}

\author{
Drs. JUAN PABLO QUINTEROS P. ${ }^{1}$, JUSSARA SOUSA R. ${ }^{1}$, \\ Int. ANDREA RAPIMAN G. ${ }^{2}$, FABIO VALDÉS G. ${ }^{1}$ \\ 1 Unidad de Anatomía Normal. \\ 2 Interna de Medicina. \\ Facultad de Medicina, Universidad de Los Andes. \\ Santiago, Chile.
}

\begin{abstract}
Anatomical description of the blood supply of the distally based sural flap in eight lower limbs

Background: The distally based sural fasciomyocutaneous flap is widely used in the coverage of deep soft tissue defects on the distal third of lower limbs. Aim: To perform a morphometric description of the perforating arteries that supplies the flap. Material and Methods: We used eight lower limbs from amputations performed above the knee. The limbs were injected through the popliteal artery with red colored latex. After the injection, the limbs were dissected to obtain a distally based sural fasciomyocutaneous flap. Once the fasciomyocutaneous island was obtained, the flap was elevated dissecting its pedicle. Once the whole flap was dissected, a morphometric registry of the lateral and medial perforating arteries was performed. The pivot point for the flap was set $5 \mathrm{~cm}$ above the upper border of the lateral malleolus. The distance between the upper border of the lateral malleolus and the emergence of each perforating artery was measured. Results: The sural nerve was identified in all eight dissections. A perineural plexus was the source of the blood supply of the flap, in three of the eight dissections. In two dissections, three sural arteries were identified (medial, median and lateral). The lateral sural artery was identified in two dissections and the medial and lateral arteries in one. Three to six perforating arteries were identified in the medial part of the pedicle and four to five perforating arteries in the lateral part of the pedicle. Conclusions: The distribution of the sural artery along the flap's pedicle is very variable. The most common distribution in these dissections was in the form of a perineural plexus. Considering the distance from the lateral malleolus to the emergence of the perforating arteries, the pivot point of the flap, should be set approximately at 5.5 centimeters above the lateral malleolus.
\end{abstract}

Key words: Surgical flap, sural nerve, anatomy.

\section{Resumen}

El colgajo sural fasciomiocutáneo es ampliamente utilizado en la reparación de defectos profundos de tejidos blandos del miembro inferior distal. Este estudio describe su base anatómica mediante la morfometría de las arterias perforantes en una muestra de nuestra población chilena. Material y Método: Se utilizaron 8

*Recibido el 26 de mayo de 2011 y aceptado para publicación el 11 de octubre de 2011.

Correspondencia: Dr. Juan Pablo Quinteros P.

Avda. San Carlos de Apoquindo 2200. Santiago, Chile.

jpquinteros1984@gmail.com 
miembros inferiores de amputaciones supracondileas. Previa repleción con látex coloreado vía poplítea, se procedió a disecar los miembros inferiores para así obtener un colgajo fasciomiocutáneo sural de pedículo distal. Una vez obtenida la isla, se procedió a elevar el colgajo y disecar su pedículo. Luego se realizó la morfometría de las arterias perforantes tanto por lateral como por medial al pedículo, desde el punto pívot definido a 5 cm cefálico al maléolo lateral, hasta la base de la isla fasciomiocutánea. Se describió la distribución de la irrigación y se realizó registro fotográfico de los hallazgos. Resultados: El paquete vasculonervioso sural con un nervio fue identificado en todas las disecciones. La morfología arterial predominante fue la distribución como plexo perineural. Se reconocen tres arterias surales (lateral, mediana y medial). Fueron identificadas 3 a 6 perforantes hacia medial y 4 a 5 hacia lateral del pedículo. Conclusiones: La distribución de la arteria sural es variable y en la mayoría de los casos se presenta como plexo perineural. Dados los hallazgos de las perforantes, consideramos que el punto de giro del colgajo se encuentra aproximadamente a 5,5 cm del maleolo lateral, lo cual coincide con el punto ideal para la viabilidad del colgajo informado en otras series.

Palabras clave: Colgajo quirúrgico, nervio sural, anatomía.

\section{Introducción}

Las lesiones de tejidos blandos en la región distal de la pierna y pie han sido siempre un problema para los cirujanos plásticos por la escasa distensibilidad de la piel, las prominencias óseas de apoyo y los resultados poco favorables obtenidos con colgajos libres. En 1981, Ponten, introdujo el concepto de colgajos fasciocutáneos siendo una buena opción en la reconstrucción de la región inferior de la pierna ${ }^{1}$.

El colgajo fasciocutáneo sural de base distal fue introducido en 1983 por Donski y Fogdestam, quienes describieron la anatomía ofreciendo con detalles la descripción del procedimiento quirúrgico y la experiencia con tres casos clínicos² ${ }^{2}$ Luego de este reporte, pasó un largo período en que no se mencionó este colgajo en la literatura, hasta 1992, año en que Masquelet lo reintroduce con una completa y precisa descripción de la anatomía relevante ${ }^{3}$. De particular importancia para nosotros es la descripción de la arteria sural.

En los años posteriores al trabajo de Masquelet, el colgajo fasciocutáneo sural a pedículo distal se transformó en el pilar de la reconstrucción de la región distal de la pierna, el talón y el pie. En la década pasada, se reportaron varios cambios a la técnica para ofrecerle mayor viabilidad y solucionar las múltiples lesiones existentes ${ }^{4-7}$.

Este trabajo tiene como finalidad estudiar la irrigación del colgajo sural en piezas cadavéricas chilenas evaluando si hay variaciones en esta, en relación a la descrita por la literatura, describiendo la presencia del nervio sural, la morfología de la irrigación sural y el número de arterias perforantes.

\section{Material y Método}

Se utilizaron 8 miembros inferiores, 5 izquierdos y 3 derechos, congelados obtenidos de amputaciones supracondíleas, del stock del pabellón de anatomía normal de la Universidad de los Andes entre los años 2007 y 2009. Dichos miembros fueron inspeccionados para evaluar la indemnidad de la región de interés. Se disecó la fosa poplítea con el fin de lograr aislar la arteria poplítea, la cual se canuló y lavó con abundante agua tibia para eliminar la sangre residual en el lecho vascular. Se procedió a la repleción arterial anterógrada con látex natural coloreado rojo con tinta china marca Rotring ${ }^{\circledR}$.

Para la obtención del colgajo se propuso un protocolo de disección, basado en la revisión de la literatura en cuanto a la descripción del colgajo.

Protocolo de Disección desarrollado por los autores en base a los trabajos de Donsky, LeFourn y Follmar.

1. Identificar los dos puntos de referencia anatómicos para la obtención del colgajo:

a. Borde proximal de la isla fasciomiocutánea localizado a $2 \mathrm{~cm}$ del pliegue poplíteo en la porción mediana de la región sural, teniendo como punto de referencia la cabeza de la fíbula.

b. Punto pívot ubicado a 5 cm superior al maléolo lateral, desde el punto palpable más inferior del maléolo, en la línea media de la pierna.

2. Obtención de isla elipsoidea de 12 a $23 \mathrm{~cm}$. de eje longitudinal por 8 a $16 \mathrm{~cm}$. de eje horizontal (Figura1).

3. Identificar el eje neurovascular.

4. Elevar la isla fasciomiocutánea del colgajo.

5. Disecar la piel desde el borde inferior de la isla hasta el punto pívot, respetando el pedículo.

6. Levantar el pedículo subcutáneo distal a la isla, de $2 \mathrm{~cm}$ de ancho, incluyendo el eje neurovascular, hasta el punto pívot, identificando y disecando las arterias perforantes durante el trayecto.

Una vez elevado, completada la disección, se realizó una descripción morfométricamente los vasos perforantes, utilizando como instrumento un caliper de precisión de 150 mm (Mituyoyo®, precisión 


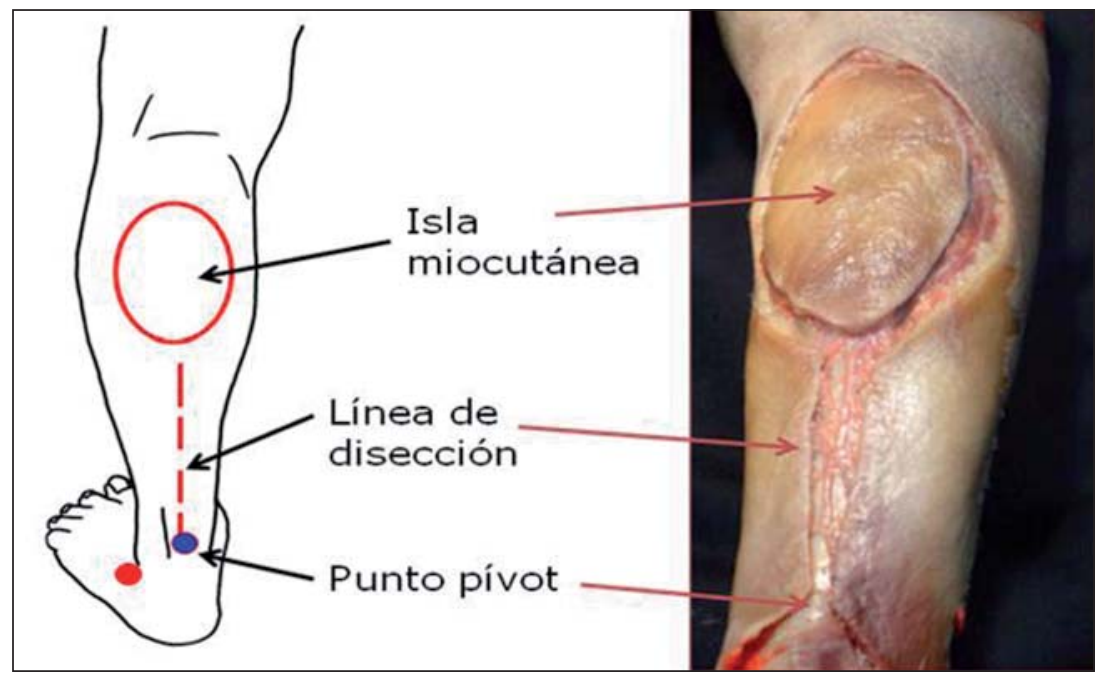

Figura 1. Disección.

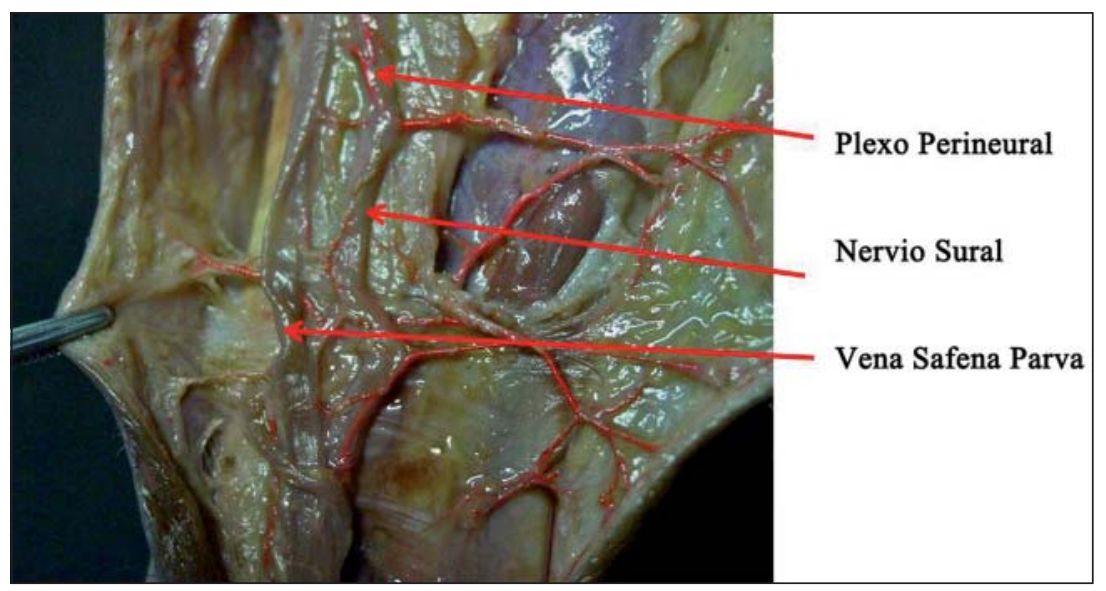

Figura 2. Plexo perineural.

0,05 mm). Se midió el punto de emergencia desde la arteria fuente en relación al maléolo lateral, desde proximal hacia distal, tanto por lateral como medial. Se hizo registro fotográfico de dichas perforantes.

\section{Resultados}

Se obtuvo un total de 8 miembros inferiores, de sexo desconocido, 5 izquierdos y 3 derechos. Dichos miembros presentaban múltiples lesiones consecuencia de patología arterial. Pese a esto se logró repleción completa en los 8 miembros.

El paquete vasculonervioso sural con un nervio fue identificado en todas las disecciones. La morfología arterial predominante fue la distribución como plexo perineural (Figura 2). Otras formas de presentación fueron la presencia de tres arterias surales (la- teral, mediana y medial) y variantes en la presencia de dichas arterias como se describe en la Tabla 1.

Se identificaron de 3 a 6 perforantes hacia medial y de 4 a 5 hacia lateral del pedículo. La primera perforante se encuentra a 5,3 cm y 5,1 cm. promedio del maléolo lateral, hacia medial y lateral respectivamente (Tablas 2 y 3 ).

Tabla 1. Resultado de la disección, distribución de la arteria sural

\begin{tabular}{|lc|}
\hline Hallazgo & $\begin{array}{c}\text { n de miembros } \\
\text { inferiores }\end{array}$ \\
\hline Plexo perineural & 3 \\
\hline Arteria sural lateral & 2 \\
\hline Arteria sural lateral y medial & 1 \\
\hline Arteria sural lateral mediana y medial & 2 \\
\hline
\end{tabular}


DESCRIPCIÓN ANATÓMICA DE LA IRRIGACIÓN DEL COLGAJO SURAL A PEDÍCULO DISTAL EN PIEZAS ...

Tabla 2. Descripción y análisis de vasos perforantes

\begin{tabular}{|lcc|}
\hline & Mediales & Laterales \\
\hline Número máximo de perforantes & 6 & 5 \\
\hline Número mínimo de perforantes & 3 & 4 \\
Promedio perforantes & 4 & 4 \\
Mediana perforantes & 5 & 4 \\
\hline
\end{tabular}

Tabla 3. Distancia en $\mathrm{cm}$ de la primera perforante (de distal a proximal) en relación al maléolo lateral

\begin{tabular}{|lcc|}
\hline & Medial & Lateral \\
\hline Máximo & 6,6 & 7,4 \\
Mínimo & 1,5 & 1,0 \\
\hline Promedio & 5,3 & 5,1 \\
\hline Mediana & 5,9 & 5,5 \\
\hline
\end{tabular}

\section{Discusión}

La importancia que ha adquirido en la cirugía reconstructiva el colgajo sural a pedículo distal por su capacidad de cubrir lesiones distales de pierna y pie, además de ser fácil de reproducir, se debe también a los estudios anatómicos que se han realizado a nivel mundial sobre la vasculatura que nutre este colgajo fasciomiocutáneo, describiendo la distribución de la arteria sural como variable y en la mayoría de los casos como plexo perineural. Un ejemplo de esto es el estudio anatomoangiográfico de Aoki S. y los resultados obtenidos en este estudio ${ }^{8}$.

La presencia de perforantes del plexo arterial perineural sural hacia el músculo gastrocnemio, la comunicación existente con la irrigación arterial de la piel y la prevalencia de este plexo en la población determinan una importante función en la vitalidad del colgajo sural ${ }^{7}$. Trabajos como los de Aoki S. demostraron que el plexo arterial perineural sural es el más importante en la supervivencia del colgajo sural a pedículo distal y para maximizar la viabilidad del colgajo se deben incluir: nervio sural, arteria sural y vena safena parva ${ }^{4,8}$.

En la mayoría de las piezas estudiadas en el presente trabajo, el plexo arterial perineural se presenta apoyando lo descrito en la literatura. Dados los hallazgos de las perforantes, concluimos que el punto de giro del colgajo se encuentra aproximadamente a $5,5 \mathrm{~cm}$ del maléolo lateral, lo cual coincide con el punto ideal para la viabilidad del colgajo informado en otras series ${ }^{2,9,10}$.

Dentro de las limitaciones del trabajo está la dificultad de obtención de piezas anatómicas -lo cual determinó un tamaño muestral pequeño-, el desconocimiento del motivo de amputación, la presencia de patología vascular en su mayoría Diabetes Melli- tus. Sin embargo, se logró una repleción exitosa y una clara visualización de la microvasculatura a la disección, tal como lo describe el estudio Malokov, de 24 amputaciones con enfermedad vascular severa, el colgajo era teóricamente posible en 23 piezas ${ }^{11}$.

Como conclusión de este estudio anatómico, la irrigación del colgajo sural a pedículo distal realizado en piezas cadavéricas chilenas se correlaciona con lo descrito en la literatura a nivel mundial.

\section{Agradecimientos}

Dr. Alex Eulufí Muñoz por su colaboración con el desarrollo del trabajo.

\section{Referencias}

1. Ponten B. The fasciocutaneous flap: its use in soft tissue defects of the lower leg. Br J. Plast Surg. 1981;34:21520.

2. Donski PK, Fogdestam I. Distally based fasciocutaneous flap from the sural region. A preliminary report. Scand J Plast Reconstr Surg. 1983;17:191-6.

3. Masquelet AC, Romana MC, Wolf G. Skin island flaps supplied by the vascular axis of the sensitive superficial nerves: anatomic study and clinical experience in the leg. Plast Reconstr Surg. 1992;89:1115-21.

4. Wong $\mathrm{CH}$, Tan BK. Maximizing the reliability and safety of the distally based sural artery flap. J Reconstr Microsurg. 2008;24:589-94.

5. Reyes S, Andrades P, Fix RJ, Vasconez L. Distally based superficial sural fasciomusculocutaneous flap: a reliable solution for distal lower extremity reconstruction. J Reconstr Microsurg. 2008; 24:315-22.

6. Thione A, Valdatta L, Buoro M, Tuinder S, Mortarino C, Putz R.The medial sural artery perforators: anatomic basis for a surgical plan. Ann Plast Surg. 2004;53:250-5.

7. Yu AX, Deng K, Tao S, Yu G, Zheng X. Anatomic study and clinical application of distally-based neuromyocutaneous compound flaps in the leg. Microsurgery 2007;27:528-32.

8. Aoki S, Tanuma K, Iwakiri I, Mizuno H, Ogawa R, Ozawa H, et al. Clinical and vascular anatomical study of distally based sural flap. Ann Plast Surg. 2008;61:738.

9. Le Fourn B, Caye N, Pannier M. Distally based sural fasciomuscular flap: anatomic study and application for filling leg or foot defects. Plast Reconstr Surg. 2001;107:67-72.

10. Follmar KE, Baccarani A, Baumeister SP, Levin L, Erdmann D. The distally based sural flap. Plast Reconstr Surg. 2007;119:138-48.

11. Malokov S, Casa Nova D, Magalon G, Branchereau, A. Sural flap vascularization in arteritic patients: An anatomic study of 24 amputation specimens. Surg. Radiol Anat. 2003;25:372-8. 